\title{
Aerobic capacity for breast cancer survivors 2 to 3 years after breast surgery
}

\author{
Rudolfs Ceseiko, ${ }^{1, *}$, Signe Tomsone ${ }^{1}$, Laura Majevska ${ }^{1}$, and Aivars Vetra ${ }^{1,2}$ \\ ${ }^{1}$ Rīga Stradingš University, Faculty of Rehabilitation, Riga, Latvia \\ ${ }^{2}$ Riga Eastern University Hospital, Riga, Latvia
}

\begin{abstract}
Breast cancer treatment may cause unfavourable side effects that directly or indirectly influence the cardio-respiratory capacity among breast cancer survivors, thus affecting physical functioning and the quality of life. Mounting evidence supports that Maximal oxygen uptake $\left(\mathrm{VO}_{2 \max }\right)$ is a strong predictor of all-cause mortality and cardiovascular disease, and low $\mathrm{VO}_{2 \max }$ is associated with increased mortality from breast cancer. The aim of the study was to measure cardio-respiratory fitness in breast cancer survivors and determine to what extent $\mathrm{VO}_{2 \max }$ levels are lower compared with healthy counterparts from population. The study included 11 women with a history of breast cancer, the average time after breast surgery being 2-3 years. Aerobic capacity was determined by the Cardiopulmonary exercise test. HUNT3 study was used to define aerobic capacity for healthy women in population. Mean Age for participatns $50 \pm 4$ years $(\mathrm{M} \pm \mathrm{SD})$. The comparison of the results from Cardiopulmonary exercise test indicated that breast cancer survivors display $\mathrm{VO}_{2 \max }$ values significantly lower $27.1 \mathrm{ml} / \mathrm{kg} / \mathrm{min}(27 \%)$ than healthy counterparts from population, i.e., $34.4 \mathrm{ml} / \mathrm{kg} / \mathrm{min}(P=0.001)$. Recognizing the increasing number of breast cancer patients worldwide and the physical reconditioning among the survivors, aerobic exercise training is suitable for improving cardiorespiratory health, functionality, and quality of life.
\end{abstract}

Key words: breast cancer, rehabilitation, aerobic capacity.

\section{Introduction}

Breast cancer (BC) is the most common type of cancer among women. In 2012, about 1.7 million new cases were diagnosed worldwide, thus representing $25.2 \%$ in morbidity rates and $14 \%$ in mortality rates from the overall cases of oncologic diseases among women [1]. Every year more than 1000 new cases of $\mathrm{BC}$ are diagnosed in Latvia. At the end of year 2016, more than 12,000 BC patients had been registered. After the diagnosis, about $89 \%$ of the patients live more than 5 years [2]. The current methods for treatment of oncologic diseases imply long combined local and/or systematic therapy. It initiates various acute and toxic manifestations leading towards increased risk of mortality and chronic diseases not primarily associated with the diagnosis of cancer. The decrease in the values of mortality and quality of life are mainly associated with cardiovascular and cardiopulmonary diseases [3] having huge effect on the overall participation in daily activities and increased rates

\footnotetext{
* Corresponding author: Rudolfs. Ceseiko@rsu.lv
} 
of disability [4]. Aerobic capacity is the most objective indicator of the functional condition of the cardiovascular and respiratory systems. Aerobic capacity characterizes the possibilities of using oxygen transport in skeletal muscles. Reduced aerobic capacity rates can be explained by several variables, e.g., pulmonary, cardiovascular and peripheral. Aerobic capacity is determined by the results from the maximum or sub-maximal exercise test performed under laboratory conditions with appropriate equipment [5].

Long-term complications after cancer treatment are associated to the dysfunction of specific organ systems, resulting in various chronic diseases, functional restrictions (for instance, lymphodema), specific pain syndromes, neuropathy, secondary malignant tumours and aesthetic defects. All of the mentioned side effects are mostly typical to patients younger than 60 years and those having suffered from chronic diseases (diabetes, hypertension, etc.), or patients being exposed to infectious diseases or recent surgical interventions [4]. BC patients are exposed to nearly three times higher risk of developing one of the cardiovascular diseases in comparison to healthy counterparts [6]. In most of the postmenopausal BC patients, at least one of the risk factors for cardiovascular disease is identified already at the time of cancer diagnosis [7]. The cardio-toxic side effect derived from adjuvant therapy has the potential of significantly increasing mortality rates among BC patients diagnosed with cardiovascular diseases. Anthracycline medicine causes immediate damage of myocardial cells while clinically relevant changes can be noticed only after several months or years. Acute cardio-toxicity during treatment period is very rare, while subacute or chronic myocardial damage (within a year after treatment) is noticed in about $23 \%$ of cases. Similar side effects are caused also by hormone therapy, which increases triglyceride levels, thus leading towards increased risk of atherosclerosis [8]. Radiation therapy can cause local damage of myocardial walls, resulting in decrease in myocardial perfusion [9].

The cardiovascular system is especially sensitive to the reduction in physical activities, thus even periods of short-term physical inactivity or bed rest causes significant decrease in aerobic capacity for both physically active and inactive population [10]. The decrease in physical activities among BC patients is observed at the beginning of chemotherapy. The entire treatment process lasts for 9 months or longer; in this period physical activity is reduced to minimum or excluded completely. After 4 weeks of chemotherapy, $\mathrm{VO}_{2 \max }$ decreases for about 3-8\% [11]. The studies show that $\mathrm{VO}_{2 \max }$ reaches $25 \mathrm{ml} / \mathrm{kg} / \mathrm{min}$ in average prior to adjuvant therapy. That is by $17 \%$ lower than among healthy, yet physically inactive women. However, the maximum oxygen consumption after the adjuvant treatment is about $22.2 \mathrm{ml} / \mathrm{kg} / \mathrm{min}, 25 \%$ lower than in healthy women of the same age [9]. To be of complete functional independence, the minimum $\mathrm{VO}_{2 \max }$ value has to be at least $15.4 \mathrm{ml} / \mathrm{kg} / \mathrm{min}$. It is surprising that $30 \%$ of the patients are below this value, which indicates huge effort needed to perform daily activities. As a result, higher energy is needed and the sense of fatigue becomes present, which is very typical for BC patients undergoing treatment [12]. Decrease in the value of $\mathrm{VO}_{2 \max }$ for $\mathrm{BC}$ patients is present even for approximately 7 years after treatment [9].

Thus, the aim of the study is to measure aerobic capacity for breast cancer patients having undergone breast surgery 2-3 years ago, and compare these results with the literature data about healthy and physically inactive women of the same age.

\section{Methods}

\subsection{Participants}

The selection of the subjects was based on the following inclusion/exclusion criteria. Inclusion criteria were: breast cancer stages I-III, breast surgery done 2-3 years ago, 
40 to 55 years old, body mass index ranging from 22 to 28 , not using beta blockers, were undergoing chemotherapy, radiotherapy and hormone therapy, at least a year has passed since the last chemotherapy, not engaged in regular, targeted physical activities on a daily basis. Exclusion criteria were: breast cancer recurrence 2-3 years after the surgery, Chronic Obstructive Pulmonary Disease (COPD), bronchial asthma, hypertension, arrhythmia, heart failure, ischemic heart disease or orthopaedic disease. The study was conducted in Riga Stradinšs University, Faculty of Rehabilitation, in corporation with research laboratory of Rehabilitation. The data was collected between January $9^{\text {th }}$ and February $10^{\text {th }}, 2018$. The selection of research subjects was performed in corporation with cancer patient support society "Dzīvības koks" ("Life Tree"). Each participant received a letter with information about the study beforehand. Informed consent was obtained from all the participants. Before engaging in the study, the subjects were reached by phone, to explain testing details. When arriving to the assessment procedure, the subjects had to take with them comfortable clothing and footwear, as well as the statement from the medical treatment institution confirming information on the surgery and treatment received. The study was conducted in accordance with the ethical standards - the study was authorised by the Ethics Committee of Riga Stradiņš University and followed the principles set in Declaration of Helsinki.

To evaluate aerobic capacity, incremental treadmill test was applied to measure $\mathrm{VO}_{2 \max }$ [13]. The test was performed by the use of treadmill Mercury ( $h / p /$ cosmos, NussdorfTraunstein, Germany), heart rate monitor Polar (F7, Finland) and spiroergometer Cortex MetaMax 3B (Cortex Biophysik, Leipzig, Germany). On the day of the test, the spiroergometer was calibrated according to the manufacturer's instructions. A short history of diseases and the body weight of each participant were collected prior to the beginning of the testing. The participants were introduced with the study procedure. They were engaging in a 5-minute long warm-up session on a treadmill at a self-selected speed. A modified Balke protocol was used for measuring $\mathrm{VO}_{2 \max }$ [14]. The maximum expected heart rate was calculated on the basis of formula 220-age (in years) [15]. The heart rate was monitored at the beginning of the test and during the last 30 seconds of it. Afterwards, incremental test on a treadmill was performed, starting with constant speed of $4.8 \mathrm{~km} / \mathrm{h}$ and an incline of $0 \%$. The intensity was gradually increased by lifting the treadmill slope by $2 \%$ every minute until 20\% level is reached. The test was interrupted under the following conditions: $90 \%$ of the maximum heart rate is reached; $\mathrm{VO}_{2 \max }$ stops increasing (reaching plateau); limiting symptoms emerged and the participant demanded the test to be interrupted or failed to respond to repeated incentive to continue. The test was performed once for each participant and the whole testing procedure was supervised by the laboratory personnel.

The data on $\mathrm{VO}_{2 \max }$ value in healthy, physically inactive women was obtained from HUNT3 study - the most prominent collection of data on the results from maximum load test among the population of Europe. Totally 428 women at the age between 50 to 59 years were evaluated. The mean $\mathrm{VO}_{2 \max }$ for this group is $34.4 \pm 5.7 \mathrm{ml} / \mathrm{kg} / \mathrm{min}$ [16].

\subsection{Statistics}

The statistical processing of data was performed by computer programme IBM SPSS. GraphPad Prism was applied for creating graphics. The method of descriptive statistics was used for group characteristics (all values being expressed as mean \pm standard deviation). One sample $\mathrm{T}$ test was applied for comparing $\mathrm{VO}_{2 \max }$ value for the group with the mean value for the population of the same age and gender as described in the literary sources. The result was considered statistically significant if $P<0.05$. 
Table 1. Participant characteristics. Breast cancer stage: number of patients with diagnosis. Therapy received: number of patients having received specific treatment.

\begin{tabular}{ll}
\hline & $(\mathbf{N}=\mathbf{1 1})$ \\
\hline Age & $50 \pm 4$ \\
Weight, $\mathrm{kg}$ & $75 \pm 17$ \\
Height, cm & $168 \pm 6$ \\
Body mass index & $26 \pm 5$ \\
Stage of cancer $(\mathbf{N})$ & \\
I & 2 \\
II & 7 \\
III & 2 \\
Treatment: & \\
Chemotherapy & 10 \\
Radiation & 10 \\
Hormone therapy & 10 \\
\hline Data are presented as mean $\pm \mathrm{SD}$. &
\end{tabular}

Table 2. Physiological parameters after $\mathrm{VO}_{2 \max }$ test.

\begin{tabular}{cc}
\hline & $(\mathbf{n}=\mathbf{1 1})$ \\
\hline $\mathrm{VO}_{2 \max }\left(\mathrm{mL} \cdot \mathrm{kg}^{-1} \cdot \mathrm{min}^{-1}\right)$ & $27 \pm 4$ \\
$\mathrm{VO}_{2 \max }\left(\mathrm{L} \cdot \mathrm{min}^{-1}\right)$ & $1.9 \pm 0.2$ \\
$\mathrm{~V}_{\mathrm{E}}\left(\mathrm{L} \cdot \mathrm{min}^{-1}\right)$ & $59 \pm 14$ \\
$\mathrm{HR}_{\text {max }}$ & $167 \pm 7$ \\
\hline Data is presented as mean $\pm \mathrm{SD} . \mathrm{VO}_{2 \max }:$ maximal oxygen uptake; \\
$\mathrm{V}_{\mathrm{E}}$ : ventilation; RER: respiratory exchange ratio; $\mathrm{HR}_{\max }$ : maximal heart rate.
\end{tabular}

\section{Results}

Basing on the letters sent, 21 participants applied for the study. According to the inclusion/exclusion criteria, 11 women were eligible. Patient's characteristics are presented in (Table 1). The rest of the women were not included in the study due to their age or too high body mass index.

Maximal oxygen uptake. The literary sources describing data on Cardiopulmonary exercise test among the population of Europe claim the maximal oxygen uptake for healthy women at the age of 50 years to be $34.4 \mathrm{ml} / \mathrm{kg} / \mathrm{min}$ [16]. The incremental treadmill test, performed on the research subjects $(n=11)$ resulted in the mean $\mathrm{VO}_{2 \max } 27.1 \mathrm{ml} / \mathrm{kg} / \mathrm{min}$ (Table 2). As a result, the difference $7.3 \mathrm{ml} / \mathrm{kg} / \mathrm{min}$ between the average value in patients and value existing in society is assumed to be statistically significant $(P=0.001)$ and $(95 \% \mathrm{TI}$ is from -10 to -5$)$; thus, Cohen's d value shows extra-large difference $(d=1.7)$ (Fig. 1). The results obtained are by $27 \%$ lower in comparison to the data on healthy peers from the population.

\section{Discussion}

The main finding of the present study was that aerobic capacity of BC patients is by $27 \%$ lower than that of their counterparts (healthy, physically inactive women of the same age). Several studies investigating the aerobic capacity of 50-year old BC patients have demonstrated decrease in maximum oxygen consumption by approximately $22-30 \%$ after the treatment [17]. The data indicate the negative influence (which can persist even several years afterwards) of the treatment side effects on the body's aerobic capacity in a long run. Reduced aerobic capacity can remain present even up to 7 years after diagnosis [9]. 


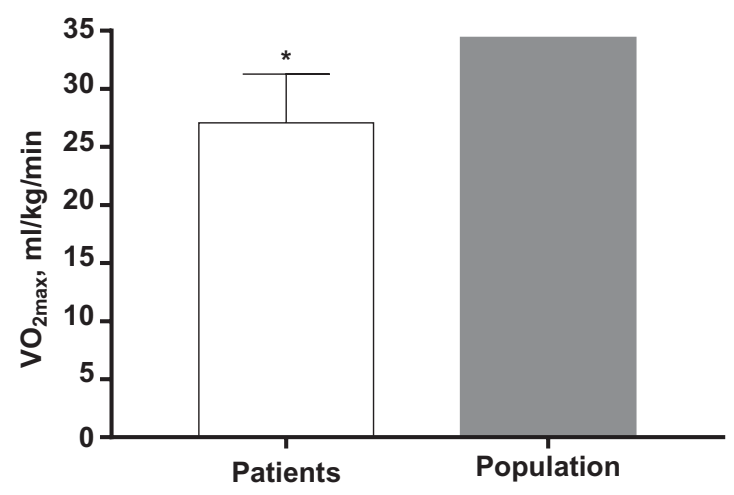

Figure 1. Maximal oxygen uptake for breast cancer patients compared with heathy counterparts from population. Data is presented as mean $\pm \mathrm{SD}, * P=0.001$.

$\mathrm{VO}_{2 \max }$ indicators depend on $\mathrm{BC}$ stage and the treatment received. Aerobic capacity will be higher for patients in the stage I or II, while the capacity will be lower in stage III or IV of the cancer. A study conducted by Jones et al. (2012) investigated BC patients, who had undergone the last chemotherapy from 6 months to 3 years ago. The average $\mathrm{VO}_{2 \max }$ was $18.4 \mathrm{ml} / \mathrm{kg} / \mathrm{min}$, while the average $\mathrm{VO}_{2 \max }$ in forth (metastatic) stage was $16.3 \mathrm{ml} / \mathrm{min} / \mathrm{kg}$. It is by $33 \%$ lower than for healthy and physically inactive women of the same age [12].

Nine of the 11 participants in the present study have been diagnosed with $\mathrm{BC}$ at the stage I or stage II. In general, the results obtained are higher in comparison to other researches devoted to aerobic capacity. However, the results greatly depend also on the testing methods of aerobic capacity. Cardiopulmonary exercise testing (CPET) mostly is applied in 2 ways, either using treadmill or veloergometer. One of the techniques is by gradually increasing the load on a veloergometer or treadmill. According to the data of various studies, $\mathrm{VO}_{2 \max }$ rates on a treadmill for $\mathrm{BC}$ patients are slightly higher than on a veloenergometer. This phenomenon could be explained by the fact that bigger number of muscle groups is involved in walking activity. It leads towards higher blood supply to the congested muscles and need for more oxygen. Even though the Cardiopulmonary exercise test demands great physical effort, it is relatively safe for BC survivors when accompanied by appropriate screening techniques [17].

Reduced $\mathrm{VO}_{2 \max }$ rates are explained by multi-factor reasons, including several organ systems engaged in oxygen transport. The limitations of cardiovascular system are the ones described most prominently in the study literature devoted to BC patients. These limitations are mostly associated with the chemotherapy medicine applied during the treatment procedure and the cardio-toxic effect caused by this medicine in a long run. Also, medical exposure causes local damage of myocardial walls [9]. As 10 out of 11 participants had been exposed to chemotherapy, local radiation or hormone therapy, and none of the participants had been suffering from cardiopulmonary diseases, it can be concluded that the effect of the treatment has influenced the results of $\mathrm{VO}_{2 \max }$ obtained during the study. 9 of 11 participants acknowledged the failure of respiratory system being the limiting factor of the incremental test. The data indicate the main limiting factors of the aerobic capacity, including cardiovascular, respiratory and circulatory systems. Since gas diffusion in the lungs is hardly a limiting factor, it can be assumed that aerobic capacity is mostly affected by the volume of cardiac output and myocardial contractility. The medicine used during the therapy does not affect the functions of the skeletal muscles, yet they can be influenced by low physical 
activities. As a result, the muscle mass decreases while the mass of fat increases. It leads towards diminished maximum muscle strength significantly limiting the aerobic capacity [2].

The mean body mass index for the study group was $26 \mathrm{~kg} / \mathrm{m}^{2}$. It suggests that $\mathrm{BC}$ patients are slightly overweight. Gain in weight during therapy and after it, body mass index being above $25 \mathrm{~kg} / \mathrm{m}^{2}$, is associated with increased mortality risk [18]. Women with extra weight $\left(\mathrm{BMI}<25 \mathrm{~kg} / \mathrm{m}^{2}\right)$ are at twice the risk of $\mathrm{BC}$ recurrence than those within the normal BMI [19].

Recently, the interest in physical activities for $\mathrm{BC}$ rehabilitation is increasing. The data available support the effectiveness of aerobic training for improving the physical condition of $\mathrm{BC}$ patients during and after the therapy. The studies suggest that the lowest physical activity among BC patients is just after finishing therapy. However, the situation stabilises after several weeks, but due to various treatment side effects and other diseases such condition can persist for a longer period. For instance, the mean $\mathrm{VO}_{2 \max }$ rate just after the adjuvant therapy is from 18.4 to $20.6 \pm 6.7 \mathrm{ml} / \mathrm{min} / \mathrm{kg}$ [2]. When experiencing severe decrease in aerobic capacity, patients find it difficult to engage in daily activities - maximum effort is required for going upstairs or working in a garden. Thus, moderate physical activities are crucial for BC patients in order to feel good, diminish the presence of the side effects of chemotherapy and limit the risk of additional diseases even before starting the therapy.

Physical activities can help in controlling body weight. It has been proved that gradual overweight reduction can contribute to better control of hypertension, hyper insularity, dyslipidaemia and pain in cancer patients [4]. Clinically relevant physical activities are those occurring after the diagnosis of BC [20]. In a study conducted by Coumeya and colleagues, $\mathrm{VO}_{2 \max }$ for $\mathrm{BC}$ patients increased by $14.5 \%$ after 17 weeks of aerobic training with a load intensity from $70 \%$ to $75 \%$ of the maximum heart rate. Also, after several weeks of training, the participants acknowledged such symptoms as fatigue, nausea and sleeping disorder having diminished [21]. According to a 24-week study performed by Scott et al. (2016) maximum oxygen uptake in BC patients increased by $32 \%$ due to aerobic training [22]. These studies verify that aerobic training in breast cancer patients do not have any negative effects on their health.

\section{Conclusion}

The present study demonstrates that aerobic capacity in breast cancer patients is greatly diminished just after the completion of therapy. Thus, effective aerobic training should be included as a part of rehabilitation procedure. However, the optimal aerobic training techniques (intensity, duration, frequency) to be performed by breast cancer patients just after the completion of the therapy have not been established yet. As the result, further clinical trials and the analysis of the obtained results are necessary for comparing different types of training methods.

\section{References}

[1] McGuire, S., World Cancer Report 2014. Geneva, Switzerland: World Health Organization, International Agency for Research on Cancer, WHO Press, 2015. Adv. Nutr. 7(2), 418-9 (2016)

[2] Klassen, O., et al., Cardiorespiratory fitness in breast cancer patients undergoing adjuvant therapy. Acta Oncol 53(10), 1356-65 (2014)

[3] Lakoski, S.G., et al., Exercise rehabilitation in patients with cancer. Nat. Rev. Clin. Oncol. 9(5), 288-296 (2012) 
[4] Aziz, N.M., Cancer survivorship research: state of knowledge, challenges and opportunities. Acta Oncol 46(4), 417-32 (2007)

[5] Helgerud, J., et al., Aerobic high-intensity intervals improve VO2max more than moderate training. Med. Sci. Sports Exerc. 39(4), 665-71 (2007)

[6] Ky, B., Priorities in the Cardiovascular Care of Breast Cancer Survivors. J. Oncol. Pract. 14(4), 205-211 (2018)

[7] Park, N.J., et al., Cardiovascular disease and mortality after breast cancer in postmenopausal women: Results from the Women's Health Initiative. PLoS One 12(9), e0184174 (2017)

[8] Bird, B.R., Swain S.M., Cardiac toxicity in breast cancer survivors: review of potential cardiac problems. Clin. Cancer Res. 14(1), 14-24 (2008)

[9] Peel, A.B., et al., Cardiorespiratory fitness in breast cancer patients: a call for normative values. J. Am. Heart Assoc. 3(1), e000432 (2014)

[10] Coyle, E.F., Hemmert, M.K., Coggan, A.R., Effects of detraining on cardiovascular responses to exercise: role of blood volume. J. Appl. Physiol. 60(1), 95-9 (1985)

[11] Courneya, K.S., et al., Effects of exercise dose and type during breast cancer chemotherapy: multicenter randomized trial. J. Natl. Cancer Inst. 105(23), 1821-32 (2013)

[12] Jones, L.W., et al., Cardiopulmonary function and age-related decline across the breast cancer survivorship continuum. J. Clin. Oncol. 30(20), 2530-7 (2012)

[13] Herdy, A.H., et al., Cardiopulmonary Exercise Test: Background, Applicability and Interpretation. Arquivos brasileiros de cardiologia 107(5), 467-481 (2016)

[14] Flo, G.L., et al., Development and Safety of an Exercise Testing Protocol for Patients with an Implanted Cardioverter Defibrillator for Primary or Secondary Indication. Cardiopulmonary Phys. Ther. J. 23(3), 16-22 (2012)

[15] Tanaka, H., Monahan, K.D., Seals, D.R., Age-predicted maximal heart rate revisited. J. Am. Coll. Cardiol. 37(1), 153-6 (2001)

[16] Loe, H., et al., Aerobic capacity reference data in 3816 healthy men and women 20-90 years. PLoS One 8(5), e64319 (2013)

[17] Neil-Sztramko, S.E., et al., Aerobic capacity and upper limb strength are reduced in women diagnosed with breast cancer: a systematic review. J. Physiother. 60(4), 189-200 (2014)

[18] Playdon, M.C., et al., Weight Gain After Breast Cancer Diagnosis and All-Cause Mortality: Systematic Review and Meta-Analysis. J. Natl. Cancer Inst. 107(12), djv275 (2015)

[19] Peel, A.B., et al., Cardiorespiratory fitness in breast cancer patients: a call for normative values. J. Am. Heart Assoc. 3(1), e000432-e00432 (2014)

[20] Ogunleye, A.A., Holmes, M.D., Physical activity and breast cancer survival. Breast cancer research : BCR 11(5), 106-106 (2009)

[21] Courneya, K.S., Exercise guidelines for cancer survivors: are fitness and quality-of-life benefits enough to change practice? Curr. oncol. (Toronto, Ont.) 24(1), 8-9 (2017)

[22] Scott, J.M., et al., Cardiovascular Late Effects and Exercise Treatment in Breast Cancer: Current Evidence and Future Directions. Can. J. Cardiol. 32(7), 881-890 (2016) 